Archives

$7 \mid 1991$

Varia

\title{
Les Tinayre, une famille de médiateurs culturels entre la France et la Hongrie
}

\section{Claude Schkolnyk}

\section{Q OpenEdition}

Journals

Édition électronique

URL : http://journals.openedition.org/ccrh/2845

DOI : $10.4000 /$ ccrh. 2845

ISSN : 1760-7906

Éditeur

Centre de recherches historiques - EHESS

Édition imprimée

Date de publication : 15 avril 1991

ISSN : 0990-9141

Référence électronique

Claude Schkolnyk, "Les Tinayre, une famille de médiateurs culturels entre la France et la Hongrie », Les Cahiers du Centre de Recherches Historiques [En ligne], 7| 1991, mis en ligne le 18 mars 2009, consulté le 19 avril 2019. URL : http://journals.openedition.org/ccrh/2845; DOI : 10.4000/ccrh.2845

Ce document a été généré automatiquement le 19 avril 2019

Article L.111-1 du Code de la propriété intellectuelle. 


\title{
Les Tinayre, une famille de médiateurs culturels entre la France et la Hongrie
}

\author{
Claude Schkolnyk
}

1 Une famille de gens simples, les Tinayre, ni universitaires, ni diplomates, des républicains auvergnats, est jetée par les allées de l'Histoire, sur la route de l'exil. Le personnage central est la mère, Victoire Tinayre, institutrice communarde, membre de l'Association Internationale des Travailleurs (A.I.T.) et romancière; elle est accompagnée de sa sœur Anna Babick et de ses cinq enfants, Julien, Louis, Caroline, Abel et André. L'aîné a 14 ans et le benjamin, 5 ans. La patrie d'adoption est, pour des raisons mal connues, la Hongrie où chacun va tenter de survivre, dans des lieux différents, en s'intégrant à son milieu d'accueil. Ainsi commence, grâce à cette dispersion, à se composer, pour le grand bonheur de l'historien, un fonds très important de correspondance échangée entre les membres de cette famille, d'une ville à l'autre, d'un milieu social à l'autre, au hasard des déplacements et des engagements. L'échange de lettres s'interrompt le temps de rares retrouvailles, mais il reste toujours un absent à qui on l'on rapporte une version des faits. A travers le regard des enfants comme celui des adultes, à travers les préjugés d'individus appartenant à une classe sociale, à une culture, à une époque, se révèle une certaine Hongrie. A cette subjectivité propre à ceux qui ont écrit ces lettres, s'ajoute celle des descendants qui ont opéré le tri et la conservation, et celle du chercheur. Malgré cela, l'étude de cette correspondance inexploitée jusqu'alors, à laquelle s'ajoutent des souvenirs écrits au crépuscule de la vie, des romans inspirés par le séjour et des documents retrouvés qui corroborent les faits, permet une approche originale et intimiste de lieux, de faits, de gens représentatifs de la Hongrie de la fin du XIX ${ }^{e}$ siècle.

2 Avant de suivre le périple de chacun et de voir comment il restitue une image de la société hongroise, il faut s'interroger sur ce choix de la Hongrie que rien en apparence ne laisse présager. 


\section{Quelques hypothèses}

3 Rien ou peu de choses: une tante maternelle de Victoire Tinayre avait bien épousé un Hongrois, Janos Pàll ${ }^{1}$. L'homme s'était intégré, semble-t-il, à la population auvergnate et n'avait plus de liens avec sa patrie d'origine; son influence n'apparaît pas déterminante. Victoire Tinayre, issue d'un milieu socialiste, participe aux grandes mutations du mouvement ouvrier en France. Elle admire Fourier, Ledoux, Proudhon. Elle milite au sein de l'A.I.T., crée elle même une association de consommateurs - caisse de secours, adhère à l'Internationale aux côtés de son ami Varlin, l'organisateur de la chambre syndicale des relieurs, et fréquente assidument les féministes Paule Minck, Andrée Léo, Eugénie Pierre et Nathalie Lemel. Elle participe ensuite dans la Commune de Paris, à la commission de l'Enseignement dirigée par Édouard Vaillant et à l'Union des Femmes organisée par l'égérie de Karl Marx, Elisabeth Dmitrieff.

4 C'est grâce à la question du travail des ouvrières qu'elle fait la connaissance du Hongrois Léo Frankel chargé de la commission du Travail et de l'Échange. La rencontre avec cet autre Hongrois aura beaucoup plus de conséquences. Léo Frankel, juif hongrois d'origine allemande, était venu se joindre à l'Association Internationale des Travailleurs de Paris en 1867, à la même époque que Victoire Tinayre; il avait pris très vite un grand ascendant sur ses membres par son expérience acquise dans le mouvement ouvrier allemand, sa connaissance des théories de Lasalle, et ses relations épistolaires avec Marx. Il conquiert l'estime des femmes de la Commune, et en particulier celle de Victoire Tinayre et d'Andrée Léo, par ses idées très avancées sur l'égalité des sexes et les droits de la femme au sein de l'émancipation ouvrière ${ }^{2}$. Une grande amitié naît entre l'Auvergnate et le jeune Hongrois «qu'elle avait pris tout d'abord pour un Prussien $»^{3}$. Pendant de nombreuses années, et sans doute jusqu'à la mort de ce dernier, Victoire Tinayre et sa soeur restèrent en liaison étroite avec lui. De là à supposer que l'institutrice ait eu des contacts avec le mouvement ouvrier hongrois reconstitué par Frankel à sa sortie des geôles autrichiennes en $1876 \ldots$ il n'y a qu'un pas. Au cours de la semaine sanglante qui suivit la débâcle de la Commune, Victoire Tinayre et son frère Jean, impliqué lui aussi en tant que directeur de la manufacture des tabacs de Reuilly, fuient en Suisse avec la plus grande partie des proscrits. Ils sont bientôt rejoints par la tante Anna et les enfants. Genève n'est pour eux qu'un lieu de passage avant la Hongrie. Des indices laissent supposer que l'Internationalisme ouvrier joua peut-être un rôle dans le choix de cette nouvelle terre d'exil. En effet, la journaliste hongroise Martha Nyilas ${ }^{4}$ attire l'attention sur le rôle joué par le rédacteur de la revue Hon, Gyorgy Aladar. Celui-ci avait fait ses études avec Édouard Vaillant en 1867 à l'Université de Tübingen, et lors d'un voyage à Londres, peu après la Commune, il s'était lié d'amitié avec Flourens. Il avait aussi rencontré Marx dont il avait fait connaître les idées dès son retour à Budapest dans plusieurs numéros du journal, parus en octobre 1871. La manifestation des ouvriers hongrois en signe de deuil, en juin 1871, après la répression sanglante des Versaillais, avait en effet suscité la curiosité des lecteurs au sujet de la Commune et de l'Association Internationale des travailleurs. D'après M. Nyilas, G. Aladar et sa femme, directrice d'école, aidèrent Victoire Tinayre à trouver du travail à Budapest.

5 Plus plausible est l'hypothèse d'un départ en Hongrie pour des raisons économiques. Genève avait été choisie comme refuge par de nombreux communards pour de multiples raisons : sa proximité avec la France, l'espoir de retrouver du travail dans une famille de 
riches étrangers en villégiature au bord du lac Léman. Malheureusement le marché est vite saturé, et des agences opportunistes voient rapidement le profit qu'il y a à mettre en contact des familles d'Europe centrale, principalement polonaises ou hongroises, avec des proscrits assez instruits pour enseigner le français, le goût parisien et l'esprit des Lumières revus par les philosophes du progrès social ${ }^{5}$. Victoire et les siens partent les uns après les autres dans des régions différentes, sur le territoire hongrois.

\section{Hongrie, terra incognita}

6 Victoire Tinayre et sa jeune sœur Anna Babick arrivent en Hongrie avec pour toute connaissance de ce pays un amalgame d'idées reçues, d'anecdotes véhiculées par le parisianisme, et de faits divers rapportés par les journaux. La Hongrie reste un pays mythique. Les récits des voyageurs de la première moitié du siècle sont fantaisistes, et les romanciers exploitent les images colorées qui plaisent au public : c'est un pays lointain et fantastique, peuplé de magnats propriétaires d'immenses domaines, de bétayrs, brigands redoutables mais patriotes et aimés du peuple, cachés dans la forêt de Bakony, de csikos parcourant la puszta à la tête de troupeaux de chevaux à demi sauvages. On y mange une cuisine épicée au paprika en buvant du Tokaj ou de la palinka et en écoutant pleurer les violons des tsiganes.

7 L'un des premiers Français à faire connaître ce pays sous un jour plus réaliste est Auguste de Gérando, neveu du philosophe et philanthrope Joseph-Marie de Gérando. Le jeune homme avait épousé la Hongrie en la personne de Emma Teleki, fille d'une des plus prestigieuses familles de la noblesse hongroise, petite-fille de Thérèse de Brunswick, filleule de l'impératrice Marie-Thérèse et « l'immortelle bien-aimée » de Beethoven ${ }^{6}$.

8 Auguste de Gérando écrivit entre autres études, La Transylvanie et ses habitants, publiée en 1845 et rééditée de nombreuses fois. Il mourut après avoir participé à la révolution hongroise aux côtés de Kossuth. Il fut le premier à développer l'idée de la «mission hongroise ", qui préconisait la liberté intérieure et l'indépendance hongroise d'une part, et d'autre part la défense des frontières qui faisaient de ce pays un état-tampon entre l'Ouest et l'Est. Entre l'oppression germanique et la pression expansionniste des Russes et des Turcs sur l'Occident. L'idée fut soutenue par les Hongrois réfugiés en France. Le journaliste Boldenyi la diffusa dans l'hebdomadaire La Hongrie ${ }^{7}$. Cette idée persista chez les hungarophiles tout au long du siècle, particulièrement chez les revanchards de 1870 (Juliette Adam la défendit bien après la publication de son livre La patrie hongroise, édité en 1884) et les détracteurs du Traité de Trianon.

9 La révolution de 1848, d'une façon générale, avait mis la Hongrie à la mode ; Victor Hugo dans son exil, Michelet, Quinet, Chassin fréquentèrent assidument les proscrits dont un grand nombre s'était installé à Paris ou à Montmorency où ils avaient formé une petite colonie autour de la famille Teleki ${ }^{8}$. Théophile Gautier portait aux nues ses amis, le dessinateur Zichy, et les peintres Madarasz ${ }^{9}$ et Munkacsy. Maupassant connaissait bien ce dernier, l'auteur adulé du «Christ devant Ponce Pilate » qu'il fit figurer dans Bel Ami ${ }^{10}$.

10 A partir de 1867, la Hongrie cessa d'être considérée comme une province autrichienne pour devenir aux yeux de l'opinion française un pays à part entière. Une étude systématique du journal Le Temps montre que l'évolution des moyens de communication, de la technologie et de l'économie donne au pays une toute autre dimension; il cesse d'être aussi fantasmagorique et y gagne en réalité. En même temps, il perd de son 
charme ; les romanciers cherchent ailleurs leur inspiration et le public tourne sa curiosité vers l'Afrique ou l'Asie lointaines, objets de leurs préoccupations politiques et militaires.

\section{Les Tinayre et la Hongrie}

11 Le premier des Tinayre à prendre le chemin de l'exil hongrois au printemps 1873 est le fils cadet, Louis Tinayre, âgé de douze ans. Il est accueilli par les propriétaires d'un domaine agricole, les Adler, juifs d'origine allemande. Ils habitent à Forro-Encs, au bord de la Hernad, près de Kassa (aujourd'hui Kosice) « la plus belle ville de la haute-Hongrie » ${ }^{11}$, capitale d'un des plus riches comitats. Sa sœur Caroline est envoyée à Nagy-Karoly, proche de Debrecen, à l'Est, dans la famille du Dr Grünhut, peu de temps après. Leur mère décide de venir à son tour et de réunir toute la famille sur le même sol. Elle fait venir sa jeune sœur, accompagnée des deux derniers enfants, Abel et André, et lui trouve un emploi de gouvernante chez la comtesse Karolyi, à Nagy-Karoly, et reste elle-même le temps d'une année scolaire pour enseigner le français aux petites filles Grünhut avant d'aller s'installer à Kassa, à proximité de son fils cadet. Cette ville de " 20.000 âmes » plus importante et plus riche, possède plusieurs écoles primaires dont une école Froebel, un gymnasium et une école royale, reale iskola ainsi qu'un institut d'agriculture. La noblesse et la grosse bourgeoisie qui y possèdent des hôtels particuliers recherchent du personnel français. Quatorze professeurs de français, dont on ne sait pas s'ils sont d'anciens communards, occupent déjà la place quand Victoire Tinayre vient y chercher du travail au milieu de l'année 1873. La mère fait venir de Suisse son fils aîné Julien, à la fin de 1874. Elle réside jusqu'en 1877 dans cette ville qui offre des possibilités d'emploi pour elle et de bonnes écoles pour ceux de ses enfants venus la rejoindre. Cette stabilité va permettre à la famille de créer des liens solides avec des Hongrois et à l'écrivain d'étudier cette nouvelle société, de peindre certains de ses personnages types, et d'élaborer l'ébauche de futurs romans. Les trois ans passés à Kassa sont une période de réflexion et de gestation, qui donnera naissance au roman Les Méprisées, roman à deux volets dont l'un est entièrement consacré à la Hongrie.

\section{Les amis d'enfance}

Sur les bancs de l'école, les enfants se lient avec des camarades hongrois et les familles sympathisent à leur tour. Les Adler convient la mère de Louis, devenu le petit précepteur de leur fils Mano, en villégiature pour les mois d'été. Ces juifs cultivés et totalement assimilés ${ }^{12}$ les introduisent dans la société qu'ils fréquentent dans la campagne environnante ou à Kassa, des juifs comme eux, ou des hobereaux et des notables locaux. Les Tinayre deviennent amis avec le docteur Thomann de Homona, à quatre kilomètres de Encs, qui soignait ses voisins, les Adler. Les enfants Thomann ont l'âge des petits Tinayre. L'un d'eux, Istvan ira par la suite au gymnasium de Kassa, avec Louis. Plus tard, il sera un pianiste célèbre, élève de Liszt, puis professeur au conservatoire de Budapest ${ }^{13}$. Ils rencontrent aussi les Weisz, une famille d'artistes dont les enfants devinrent des vedettes mondialement connues. L'un des fils, Joszef, fut à douze ans, un virtuose au piano. Il succéda à Antoine Rubinstein comme directeur du conservatoire de Saint-Pétersbourg, et devint, à Berlin, l'un des chefs les plus réputés. Le cadet, sous le pseudonyme de Rezdö Berenyi ${ }^{14}$ devint peintre naturaliste, et reprit l'atelier de Munkacsy à Paris. Leur soeur, soprano, dirigea les tournées Weisz « dans les deux Amériques ». Julien donna des leçons 
pendant deux ans au fils du juge Puky. Gyula Puky était en même temps un poète et un disciple de Janos Arany, autre poète hongrois célèbre et inséparable ami de Pétöfi ${ }^{15}$.

Très vite, les deux aînés des Tinayre, Julien et Louis se révèlent doués pour le dessin. Victoire Tinayre les pousse alors vers un métier d'art. Julien est confié à un graveur de cachets de Kassa, Alexis Odön, et Louis apprend la peinture à la Reale Tanoda, école plus orientée vers la technologie que le gymnasium classique. Il étudie dans la classe du maître Bela Klimkovics, artiste moins réputé que son frère Ferenc, peintre international, mais possédant une notoriété régionale (des œuvres de lui figurent au musée de Kosice). Il est réputé particulièrement pour les sujets religieux. Il a étudié le dessin à Vienne et a été le professeur de Bela Benczur ${ }^{16}$. Dans son atelier, les élèves apprennent à gâcher les couleurs et à peindre les fonds de tableaux avant de toucher aux personnages. Louis obtient de bons résultats et son maître conseille à sa mère de l'envoyer dans une académie. Julien est aussi très apprécié pour ses talents de graveur. Le choix se pose de les envoyer se perfectionner à Munich, Vienne ou Budapest. Ce sera Budapest en 1877.

\section{L'inspiration hongroise dans l'œuvre littéraire de victoire Tinayre}

14 A Kassa, Victoire Tinayre est éloignée de toute organisation militante et ouvrière. Elle est peu informée des événements français malgré l'existence de quelques journaux (six journaux locaux et L'Illustration), et bien qu'elle reçoive de loin en loin des nouvelles d'Issoire ou de Genève, elle se replie sur elle-même. Le temps que sa famille et son travail lui laissent, elle le consacre à observer et à noter ses impressions, soit pour les confier à sa sœur, soit pour en faire la matière d'un roman feuilleton qu'elle destine à la presse. Une version remaniée servira à étoffer un roman de commande pour l'éditeur Fayard, Les Méprisées, publié en 1861 sous le pseudonyme de Jean Guétré. Il fut longtemps attribué à Louise Michel. La deuxième partie de ce roman social restitue à la fois les clichés indélébilement inscrits dans l'inconscient culturel de l'auteur et sa connaissance fraîchement acquise des réalités quotidiennes de la terre d'accueil. La socialiste a été vivement impressionnée par la misère dès son arrivée, en 1873. La famine et le choléra sévissaient dans les campagnes ${ }^{17}$ et elle-même fut prise de fièvres. Malgré la législation, les paysans étaient toujours inféodés aux seigneurs aux mœurs brutales sinon dépravées. Elle découvre aussi une population qui lui était jusqu'alors inconnue, les juifs, sur lesquels elle a des préjugés empruntés à Fourier. Les juifs hongrois, juifs assimilés, d'origine généralement germanique, ne sont en rien différents des bourgeois. Ce sont des propriétaires de domaines rachetés à la petite noblesse désargentée ou des banquiers et des industriels citadins, ou juifs orthodoxes venus de la Galicie avec leurs traditions et leur costume distinctif. Ces derniers sont de modestes artisans ou de petits commerçants de villages ou des colporteurs, beaucoup sont pauvres. Comme Louise Michel s'est penchée sur le sort des Canaques, Victoire Tinayre rêve de construire en Hongrie un grand domaine inspiré des colonies utopiques. Les paysans y seraient rendus libres et laborieux par l'instruction, et les juifs régénérés par le travail manuel. Elle échafaude avec sa famille et quelques amis des plans pour le «Grand Oeuvre». Le départ pour Budapest y met fin pour un temps. Le projet qui transparaît dans la correspondance des années d'exil se révèlera tout entier dans le roman, faute d'avoir pu être réalisé sur le sol hongrois. 


\section{Budapest}

15 Kassa était une petite ville animée par un théâtre et les notables organisaient concerts et salons littéraires. Mais les Tinayre découvrent à Budapest, une grande animation et une effervescence culturelle auxquelles ils n'étaient pas habitués. Le milieu artistique et leurs nouvelles fréquentations les entraînent dans un nouveau réseau et stimulent les talents des uns et des autres. Ils peuvent rapidement se rendre compte que les Hongrois un peu cultivés (la bourgeoisie riche quelquefois plus que les grands magnats préoccupés d'élevage et de droit) sont à l'affût de tout ce qui est français : la bourgeoisie juive qui veut égaler la gentry dans un souci d'assimilation, la noblesse progressiste pour les idées avancées, le reste des aristocrates parce que c'est de bon ton dans toute l'Europe. D'une façon générale, parce que les Hongrois voient dans la culture française un contrepoids à la culture germanique, imposée par le pouvoir de l'Autriche. Déjà, en 1840, il y avait deux théâtres français à Pest, et le voyageur Edmond de Thouvenel écrivait que l'esprit français régnait ordinairement sur les deux scènes. Il y avait vu « représenter sur la première Le Postillon de Longjumeau, et sur la seconde, Christine, d'Alexandre Dumas, traduite en vers hongrois $»^{18}$. Les Tinayre, qui possèdent au bout de quatre ans une connaissance parfaite de la langue, découvrent à travers les nombreux quotidiens ou les revues de Budapest, les publicités de la librairie française Franklin, proposant au public hongrois un grand choix d'ouvrages de notre littérature classique, ou les annonces de conférences ou de débats littéraires en français. Victoire Tinayre va volontiers écouter ses compatriotes qui sont souvent comme elle des communards. Elle se lie d'amitié avec le plus célèbre d'entre eux, Auguste Rogeard, connu pour avoir écrit Les Propos de Labénius, un pamphlet contre Napoléon III. Réfugié à Budapest après avoir été banni de Vienne, il est l'hôte de la famille de Gerando et c'est dans les salons de Mademoiselle Antonine, fille d'Emma et Auguste de Gerando, sous la protection d'un buste de la République, qu'il donne ses leçons sur la Renaissance française ou sur l'époque des Lumières devant un brillant parterre d'artistes, d'intellectuels et de parlementaires hongrois. Rogeard introduit les Tinayre dans ce milieu et ils rencontrent Liszt, accompagné de son ami le pianiste Geza Zichy, Jokai «le Hugo des Hongrois », Pulsky, l'ancien compagnon de Kossuth. Victoire Tinayre se lie avec la jeune aristocrate qui s'intéresse de très près à la pédagogie européenne. Ces liens perdurent au delà du séjour à Budapest et les deux femmes entretiennent une correspondance après le retour de la communarde en France. Lorsque Victoire Tinayre devient en 1884 la directrice des écoles du familistère de Godin, à Guise, on voit quelquefois paraître dans Le Devoir, des articles signés Antonine Teleki de Gerando ${ }^{19}$.

\section{Levain hongrois pour des talents français}

Le but principal du séjour à Budapest est l'apprentissage d'un métier d'art. Victoire Tinayre n'envisage pas de faire de ses fils de grands artistes, elle veut développer leur talent et leur donner une pratique. Pour Louis, le futur peintre, elle imagine des débouchés dans la décoration de porcelaines. Elle destine Julien à l'illustration de romans.

17 L'académie de Budapest est en mesure de répondre à cette demande. Elle a été créée en 1871, peu de temps après le Compromis pour affirmer la spécificité du talent hongrois, mais aussi pour former des professeurs aptes à développer le goût des arts dans les écoles. 
Elle donne donc accès à plusieurs formations. Une formation purement artistique que suivra finalement Louis, une formation destinée à l'apprentissage des arts décoratifs qui correspond davantage aux souhaits de Julien et une formation pédagogique pour les futurs maîtres de dessin des écoles hongroises. La multiplicité des buts de cette nouvelle académie est le germe de la division qui se réalisera quelques années plus tard. Académie d'État créée par le ministre Tivadar Pauler, elle donne la priorité à la formation pédagogique au détriment de l'enseignement purement artistique, l'une des raisons étant que la Hongrie est trop petite pour offrir des débouchés à ses artistes. L'enseignement des arts et des arts décoratifs n'étant pas séparé, le niveau de formation des artistes est bas et l'enseignement principalement axé sur le dessin. Il faut attendre la quatrième année pour se consacrer entièrement à la peinture. Les jeunes Tinayre en subissent les conséquences. Bien préparés, ils arrivent au concours avantagés, mais les querelles intestines leur sont préjudiciables. Sous la férule du directeur Keleti, il règne à l'École des Beaux-arts un académisme étouffant auquel le talent personnel de Louis se heurte fréquemment. Cependant, les deux frères acquièrent rapidement une solide maîtrise de leur métier et se font des amis solides parmi leurs aînés comme parmi leurs camarades d'ateliers.

Louis, muni d'une recommandation de Klimkovics, est chaleureusement accueilli à son arrivée par le très parisien Viktor Madarasz. La plupart des professeurs ont acquis une expérience à l'étranger, à Vienne ou à Munich, quelquefois à Paris. Beaucoup ont laissé un nom et leurs œuvres peuplent les salles des musées nationaux quand ce n'est pas internationaux. Outre Keleti, le directeur et paysagiste, Imre Greguss dirige le dessin, Szeleky le cours d'anatomie et de peinture, Halasz préside au modelage et à la sculpture, Morelli dirige la gravure sur bois, art inexistant en Hongrie avant la fondation de l'école. Certains de leurs élèves, les compagnons de Julien et de Louis, deviendront à leur tour des célébrités d'un temps ou des valeurs sûres.

\section{Par delà les frontières}

En 1879, lorsqu'ils rentrent en France, les Tinayre restent fidèles aux artistes hongrois. Julien choisit de faire venir son ami le graveur Bénédek Balint pour l'aider dans son atelier parisien. Louis, qui parle et écrit le hongrois mieux que sa langue maternelle, va distraire sa nostalgie chez Mihaly Zichy qui ouvre sa maison à tous les Hongrois de Paris.

A Paris aussi, il a parfois l'occasion de retrouver les meilleurs : Recezs qui succédera à Benczur à la direction de l'école, et Karlowszky, tous deux lauréats du prix Munkacsy, Vago, le peintre militaire, le sculpteur Zala, Szirmay, décorateur de fresques et bien d'autres venus se tremper dans le milieu cosmopolite de Paris et exposer au Salon ou dans l'une des Expositions universelles; Louis est le plus attaché à la Hongrie. A l'âge où l'on jette un regard sur le passé, il écrit ses souvenirs et retourne une dernière fois en 1934 sur les lieux de sa jeunesse. La ville lui fait fête, la revue Budapest Szemle ${ }^{20}$ signale son passage et publie une partie de ses souvenirs. Trois ans plus tard, alors qu'il expose pour la dernière fois à Paris dans son atelier du square de Port-Royal, La Nouvelle Revue de Hongrie lui consacre un long article.

21 Le retour des autres frères s'échelonne en fonction de leurs études et des possibilités offertes par l'un ou l'autre pays. Caroline, douée pour le piano, bénéficie de leçons données par Liszt en personne, grâce à une recommandation de "Mademoiselle Antonine ». Elle restera quelques années encore avec son jeune frère André auprès de la tante Babick qui espère se constituer une retraite comme gouvernante avant de revenir 
finir ses jours auprès de sa sœur et de ses neveux. A l'âge de marier les enfants, la mère et la tante ne dédaignent pas l'idée d'une alliance avec une juive hongroise riche pour Julien ou quelque bourgeois de Budapest pour Caroline. Le hasard et le positivisme auquel toute la famille s'est convertie en décident autrement. Les enfants font des mariages français avec des disciples d'Auguste Comte, sauf Julien qui épouse une romancière, la future Marcelle Tinayre.

Abel revient rapidement auprès de ses frères tandis qu'André est partagé entre sa famille parisienne et sa culture hongroise. Ce dernier hésite longtemps avant de choisir de se fixer. La carrière diplomatique lui apporte la solution sans qu'il ait à trancher. Après un bref séjour en France, il devient vice-consul de France à Budapest avant de poursuivre un destin itinérant loin des deux patries. De sa famille, il a adopté le positivisme et il prêche sa nouvelle foi auprès de chef de file du positivisme hongrois, Samuel Kun, un typographe qu'il a connu en France. Il devient d'abord membre du Cercle des Industriels français, dont le but est de resserrer les liens culturels et économiques entre les deux pays. Il publie quelques articles dans la Revue occidentale ${ }^{21}$ et devient le secrétaire de l'Église positiviste hongroise jusqu'à sa mutation en Turquie.

\section{La tradition familiale de la seconde patrie}

Avec la mort de Victoire Tinayre en 1895 et une nouvelle dispersion de la famille, on pourrait penser qu'une page est tournée et que la Hongrie n'est plus qu'un souvenir qui s'estompe. Il n'en n'est rien et c'est paradoxalement par Julien, celui qui a séjourné le moins longtemps dans le pays, que va se perpétuer le lien. Par Julien ou plutôt par sa femme Marcelle, très proche de sa belle-mère et amie complice du jeune André.

Au tournant du siècle, Marcelle Tinayre qui a été révélée par La maison du péché est un auteur au faîte de sa gloire, traduit en plusieurs langues, quand elle découvre grâce à d'Annunzio, sa consœur hongroise en écriture, Cécile de Tormay. Avec son beau-frère André Tinayre, elle décide en 1913 de traduire Emberek a kövek között (Au pays des pierres), et les deux femmes qui échangent une correspondance mais ne se rencontrent qu'en 1914, deviennent des amies. Après la chute de la Commune hongroise, Cécile de Tormay, farouche opposante au régime du comte Karolyi comme à celui de Bela Kun écrit son violent pamphlet Bujdoso Könyv (Le livre proscrit) en 1921. Marcelle l'adapte à partir d'une traduction de Paul-Henri Régnier. Lors de l'anniversaire de la naissance de Petöfi, en 1923, la romancière française s'associe aux intellectuels pour qui cette fête est une occasion de revendiquer le retour aux frontières d'avant le Traité de Trianon. Quelques années plus tard, son fils le sculpteur Noël Tinayre qui ne connaît de la Hongrie que la légende familiale, découvre en 1933, près de Yerres, le petit cimetière des Camaldules où fut enterré le cœur du prince François II de Rakoczy. Après avoir cherché vainement les héritiers du prince pour que lui soit rendu un dernier hommage, il effectue une démarche auprès des autorités pour que ce petit carré de terre hongroise sur le sol de France devienne terre hongroise pour le tricentenaire de la naissance du héros. Les années n'ont pas tiédi les sentiments hungarophiles du petit-fils de Victoire Tinayre. Son dernier projet est de mettre en relation deux artistes de grand talent, la Hongroise Piroska Szanto et un autre peintre de Camargue, Suzanne Auchan.

Les Hongrois, de leur côté, gardent le souvenir de cette famille exceptionnelle. L'anniversaire de la Commune de Paris a donné l'occasion à l'écrivain André Lazar de retrouver sa trace ainsi que celle d'autres communards et de leur consacrer des articles et 
un livre, Arpadine ${ }^{22}$. En 1976, un lecteur du Magyar Nemzet évoque des souvenirs au sujet de l'amitié entre Istvan Thomann et les frères Tinayre. Quelques mois plus tard, un autre lecteur lui répond parce qu'il a omis de parler de Marcelle Tinayre.

Ce feuilleton reste à suivre. Mais le débat des chercheurs est tout autre: pour quelle discipline exploiter une telle saga ? Est-ce de la sociologie, de l'histoire des mentalités ou de l'histoire de l'art? Quelle importance donner à l'étude microscopique d'un petit nombre d'individus et de leurs relations, sur une courte période, au sein d'un champ aussi large que celui des échanges culturels entre deux pays ? Suffit-il de réunir les expériences proches d'autres Français venus enseigner à l'étranger pour construire une série significative et en tirer une constante? L'historien, encore fasciné par ses personnages et les imbrications des réseaux relationnels, se pose toutes ces questions et reste modeste.

\section{NOTES}

1. Archives privées de la famille Tinayre, citées dans ma thèse.

2. L'article de Léo Frankel est paru dans L'Émancipation de Toulouse, du 1er janvier 1872; il est reproduit dans La Revue socialiste de mai 1952 par Théodore Beregi.

3. Lettre de Victoire Tinayre à Anna Babick, du 16 décembre 1875.

4. Martha NYILAS, Victoire Tinayre, une communarde exilée à Budapest (en hongrois), Budapest, juin 1971. Sur G. Aladar, deux titres: KOMLOS, Tegnap és ma, Budapest, 1956 et Az idö megifjuet, Budapest, 1981 (articles sur l'A.I.T.) parus dans Hon les 20, 21, 22, 25 octobre 1871).

5. Manuscrit de souvenirs de Louis Tinayre, confirmé par AD CCC Genève : Rapport adressé au duc de Broglie, 30 novembre 1874, p. 232.

6. Antoine de GERANDO, "L'immortelle bien-aimée de Beethoven », Revue de Hongrie, juin 1986 ; Christine ADRIAENSSEN, Auguste de Gerando (1819-1849) ou l'Ère des Réformes à travers les jumelles d'un témoin français engagé, Dissertation de doctorat, Vienne, mai 1984.

7. Jean KOSA, «L'opinion française et la Hongrie au siècle dernier », La Nouvelle Revue de Hongrie, mars 1940, pp. 2 et 3.

8. André LAZAR, « Les Hongrois à Montmorency », Revue de Hongrie, Budapest, Cultura, 1985, nº 5 .

9. Théophile GAUTIER, Abécédaire, Paris, Dentu, 1861.

10. Sous le nom de Markovitch, pour un tableau intitulé « Jésus marchant sur les flots ».

11. Attila de GERANDO pour la Nouvelle Géographie Universelle. La Terre et les Hommes, d'Élisée RECLUS, Paris, Hachette, 1876, 1894.

12. Sur les juifs hongrois, cf Victor KARADY, «Les juifs dans les structures de classe en Hongrie ", Actes de la Recherche en Sciences Sociales, juin 1978, $n^{\circ} 22$.

13. Aurel VARANNAI, Magyar Nemzet, 1er février 1976 et André LAZAR, Muvesze Hörteneti Ertesitö, avril 1976.

14. Cf manuscrit de Louis Tinayre. Sous le prénom de Rudolf dans le dictionnaire Benezit; on le trouve aussi à Rezsö Bereny dans le Muveszeti Lexicon, Budapest, Academiai Kiado, 1965.

15. Cf André LAZAR, op. cit.

16. Muveszti Lexicon, tome 2, p. 651.72

17. Lettre de Julien Tinayre, 17 septembre 1873.

18. Edmond de THOUVENEL, La Hongrie et la Valachie. Souvenirs de voyage et notices historiques, Paris, Arthus Bertrand, 1840. 
19. Le Devoir du 16 décembre 1883, vol. 7 : « Mabillon et les sympathies françaises en Hongrie »; à la même époque Melle de Gerando écrivait un article sur la famille Tinayre au Familistère de Guise : "A Familistère népiskolai Guise-ben, francziaorszagban» dans la revue Csalad és iskola du 15 janvier 1884.

20. Miklos SZEMRECSANYI, « Tinayre Louis magyarordagi tatozkodasarol (1872-1879) », Budapest Szemle, novembre 1934, pp. 129 à 164.

21. Revue occidentale, Rapport sur l'exercice de l'année 1890-1891, pour le Cercle d'études positivistes de Budapest. Voir au sujet du positivisme hongrois, le mémoire de D.E.A de Laurent AGUERA, Le positivisme en Hongrie et ses adeptes orthodoxes, Budapest, 1988.

22. André LAZAR, (Bajomi Lazar, Endré), Arpadine, Szépirodalmi Könyvkiado, Budapest, 1980, ch. "A Tinayre fiverek : két magyar-francia festo".

\section{AUTEUR}

\section{CLAUDE SCHKOLNYK}

Claude SCHKOLNYCK est l'auteur d'une thèse, Victoire Tinayre (1831-1895), institutrice et féministe. Du socialisme utopique au positivisme prolétaire, préparée sous la direction de Michelle PERROT et soutenue à Paris VII en novembre 1990. Elle poursuit actuellement ses travaux sur la famille Tinayre dans la perspective des relations culturelles entre la France et la Hongrie. 